\title{
ENCAPSULATION OF LEU-ENKEPHALIN IN CORE-SHELL ISOBUTYLCYANOACRYLATE - THIOLATED CHITOSAN NANOPARTICLES FOR ORAL ADMINISTRATION
}

\author{
VÍCTOR H. CAMPOS REQUENA ${ }^{A, B *}$, KAWTHAR BOUCHEMAL ${ }^{A}$, \\ CHRISTINE VAUTHIER ${ }^{A}$, GILLES PONCHEL ${ }^{A}$
}

\author{
A. University Paris-South XI, Faculty of Pharmacy, UMR CNRS 8612. 5, rue J.B. Clément. 92296 Châtenay Malabry, France. \\ B. Master in Pharmaceutical Sciences Candidate, University of Concepción, Faculty of Pharmacy, Barrio Universitario s/n, Concepción, Chile.
} (Received: November 28, 2007 - Accepted: December 22, 2008)

\begin{abstract}
The present study shows the evaluation of Leu-Enkephalin (Leu-Enk) encapsulation on core-shell isobutylcyanoacrylate/thiolated chitosan (IBCA/ChitoTBA) nanoparticles as a tool of combined system of polymers and colloidal particles for oral administration of poor absorption drugs. Leu-Enk encapsulation using anionic emulsion polymerization technique was carried out previous depolymerization of MW 400,000 $\mathrm{g} \mathrm{mol}^{-1}$ commercial chitosan, followed by thiolation of this depolymerized chitosan with Traut's reagent (2-iminothiolane), and final characterization. Later, direct quantification of the encapsulated Leu-Enk into the nanoparticles was carried out evaluating two methodologies for nanoparticles rupture solubilization with: NaOH $1 \mathrm{~N}$ and DMSO. Physico-chemical characterization of the obtained nanoparticles showed diameters in the nano-range between 28 and $46 \mathrm{~nm}$, and yields of encapsulation of Leu-Enk between 25 and $46 \%$, and recovery yields over $96 \%$ for co-polymer nanoparticles solubilization method with DMSO.
\end{abstract}

Keywords: nanoparticles, leu-enkephalin, isobutylcyanoacrylate, thiolated chitosan, thiomers, anionic emulsion polymerization.

\section{INTRODUCTION}

Nanotechnology and polymers had captivated a tremendous interest in many scientific grounds such as pharmaceutical industry and therapeutic innovation among others. Biological and synthetic polymers had been used as a powerful tool for nanoscale systems, and modified polysaccharide-coated nanoparticles are a promising tool for drug carrier systems in oral administration of poor absorption drugs in therapeutic ${ }^{1}$. Through the recent years, great development have been reached in the field of mucoadhesive polymer systems as a new tool in formulations that increase the residence time of drugs on mucosal membranes and subsequently enhance the bioavailability of poor oral absorption drugs ${ }^{2-3}$.

Leu-Enk is a low molecular peptide (fig. 1) that behaves as a potent morphinic analgesic that is currently administered by parenteral route causing both disconfort and pain in its administration. As a peptide, Leu-Enk has a poor absorption by oral route due to their susceptibility to enzymatic metabolism by aminopeptidases $^{4}$ and for his physicochemical and size properties which limit their membrane permeation ${ }^{5}$.

For its properties, such as enzymatic biodegradability, biocompatibility and non-toxicity, chitosan is recognized as a novel excipient for drug delivery systems $^{6}$. Its natural mucoadhesive properties allows design bioadhesive drug carrier systems that can bind to the intestinal muccosa, so it can improve the residence time of drugs in the intestinal lumen and so its bioavailability? ${ }^{7}$. In the last years, thiolated chitosan derivated has been developted. This thiolated polymer - so called "thiomers" - has the advantage of increase mucoadhesion based on covalent disulfide bond between both sulphydryl groups from the thiomer and from cysteine-rich subdomains of mucus glycoproteins in intestine ${ }^{8}$. In addition, thiolated chitosan has enhancing permeation properties, and antiprotease activity due to presence of thiol groups improving the paracellular absorption of macromolecules like peptides and proteins ${ }^{9}$.

Poly(alkylcyanoacrylates) had been used widely in colloidal carrier systems for their low toxicity and biodegradability, and react spontaneously with different polysaccharides like dextran and chitosan forming stables nanoparticle systems that can be used for drug encapsulation ${ }^{10}$. Isobutylcyanoacrylate (IBCA) has been extensively used in nanoparticles systems with many polysaccharides using anionic emulsion polymerization, a simple one-step reaction in aqueous media, avoiding the use of organic solvents ${ }^{11-12}$.

For succesful drug encapsulation, several parameters must be set up including the optimal point for addition of the drug into the system, the filledup capacity of the nanoparticles system for encapsulate the drug, among others. A critical point is the separation of loaded nanoparticles from the system, for a later rupture of the amphiphilic co-polymer nanoparticles to release the encapsulated drug, and then quantify directly this encapsulated fraction without loss of drug along the process.<smiles>CC(C)CC(NC(=O)C(Cc1ccccc1)NC(=O)CNC(=O)CNC(=O)C(N)Cc1ccc(O)cc1)C(=O)O</smiles>

Figure 1. Chemical formula of Leu-Enkephalin

EXPERIMENTAL

1. Materials:

Isobutylcyanoacrylate was kindly provided as a gift by Loctite (Dublin, Ireland). Chitosan MW 400,000 $\mathrm{gmol}^{-1}$, Leu-Enkephalin acetate and Lcysteine $\mathrm{HCl}$ were purchased from Fluka (Saint-Quentin Fallavier, France), 2-Iminothiolane $\mathrm{HCl}$ (Traut's reagent) was synthesized in the Department of Organic Chemistry (Biocis UMR CNRS 8076), Faculty of Pharmacy, University Paris-XI, Châtenay-Malabry, France. Acetonitrile, trifluoracetic acid and dimethylsulfoxide were HPLC grade and purchased from Fluka. Sodium hydroxide and glaciar acetic acid were reagent grade from Merck.

HPLC was made with Interchim N5C18-25QK column; Waters 1525 Binary HPLC Peristaltic Pump; Waters In-Line Degasser AF; UV Waters 2487 Dual Lambda Absorbance Detector; and Waters 717 Plus Autosampler.

Viscometer AVS400, Schott Gerate was used for chitosan molecular weight measure. Bruker MSL-400 spectrometer (Bruker Instrument Inc. Wissembourg, France) was used for ${ }^{1} \mathrm{H}-\mathrm{NMR}$ analysis. Christ Alpha 1-4 freeze-dryer (Bioblock Scientific, Illkirch, France) were used for samples lyophilization. Sulphur elemental analysis were made in a Analyzer LECO SC144 (Service central d'analyse du CNRS, Vernaison, France). Iodine titration was assayed with a Spectrophotometer UV/VIS Lambda 11 Perkin-Elmer (Norwalk, USA). $\zeta$ potential was measured by quasi-elastic light scattering with a Nanosizer ${ }^{\mathbb{B}}$ N4 PLUS (Beckman-Couter, Villepinte, France). OptimaTM Ultracentrifuge (Bekman-Coulter Instruments, USA) was used in the ultracentrifugation of nanoparticles. Scanning Electronic Microscopy (SEM) observations were performed using a LEO 1530 (LEO Electron Microscopy Inc., Thronwood, $\mathrm{NY}$ ) operating at $3 \mathrm{kV}$ with a filament current of about $0.5 \mathrm{~mA}$. Transmission Electron Microscopy (TEM) observations were assessed using a Philips EM 208 apparatus operating at $80 \mathrm{kV}$. 


\section{Methods:}

Chitosan modifications and characterization: Commercial chitosan (MW 400,000 $\mathrm{g} \mathrm{mol}^{-1}$ ) was selectively depolymerized following the method developed by Huang et al. using sodium nitrite to obtain three average molecular weights: $30,000,85,000$ and 145,000 g mol- $^{-1}$ (called Chito30, Chito 85 and Chito 145) which were measured by capillary viscosity ${ }^{13}$. ${ }^{1} \mathrm{H}-\mathrm{NMR}$ analysis were carried out for chitosan structural change and deacetylation level measures.

The inclusion of thiol groups in the different depolymerized chitosan was carried out following the method developed by Bernkop-Schnürch et al. ${ }^{14}$ reacting one gram of chitosan with Traut's reagent (2-iminothiolane) in a weight ratio of 5:2. After an incubation period of $24 \mathrm{~h}$ at room temperature under continuous stirring, the resulting thiolated polymer was dialysed (Spectra/Por ${ }^{\mathrm{B}}$ 3 membrane MWCO:3500) against different aqueous media: $8 \mathrm{~h}$ against $5 \mathrm{~L}$ of $5 \mathrm{mmol} \mathrm{L}^{-1} \mathrm{HCl}, 8 \mathrm{~h}$ against $5 \mathrm{~L}$ of $5 \mathrm{mmol}^{-1} \mathrm{HCl}$ containing $1 \% \mathrm{NaCl}$ two times, $8 \mathrm{~h}$ against $5 \mathrm{~L}$ of $5 \mathrm{mmol}^{-1} \mathrm{HCl}$, and finally, $8 \mathrm{~h}$ against $5 \mathrm{~L}$ of $1 \mathrm{mmol}$ $\mathrm{L}^{-1} \mathrm{HCl}\left(40 \mathrm{~h}\right.$ in total). Dialysed products were freeze-dried and stored at $-20^{\circ} \mathrm{C}$ until use. The resulting polymers obtained were chitosan-4-thiol-butylamidine derivated, named Chito30-TBA, Chito85-TBA and Chito145-TBA according to the original molecular weight of the corresponding unmodified polymers.

Sulphur elemental analysis and iodine titration were performed in order to determine total sulphur and reduced thiol groups contented in modified chitosan, using the same method for nanoparticles assays.

Nanoparticles preparation: Nanoparticles elaboration following anionic emulsion polymerization was carried out according to previous works ${ }^{15-17}$. Briefly $0.069 \mathrm{~g}$ of depolymerized chitosan, at proportions of chitosan:chitosanTBA 75:25 (w/w), were dissolved in $4 \mathrm{~mL}$ of $0.2 \mathrm{~mol}^{-1} \mathrm{~L}^{-1}$ nitric acid in MilliQ water, in a glass tube at $40^{\circ} \mathrm{C}$ under gentle stirring and argon bubbling. After $10 \mathrm{~min}, 1 \mathrm{~mL}$ of a $0.2 \mathrm{molL}^{-1}$ nitric acid solution, and $0.25 \mathrm{~mL}$ of IBCA were added under vigorous magnetic stirring. Argon bubbling was kept for additional $10 \mathrm{~min}$ and stopped. The reaction was allowed to continue at $40^{\circ} \mathrm{C}$ under gentle stirring for $40 \mathrm{~min}$. After cooling to room temperature, $\mathrm{NaOH} 1 \mathrm{~N}$ was added to raise the $\mathrm{pH}$ from 1.5 to 4.5 to store.

Inclusion of Leu-Enkephalin in the nanoparticles: The preparation of nanoparticles is conduced at $\mathrm{pH} 1.5$ during $40 \mathrm{~min}$ and then $\mathrm{pH}$ was raised to 4.5 to store. Stability study of Leu-Enk in acidic medium at pH 1.5 and 4.5 is required. After one week of aqueous Leu-Enk solution incubation at $\mathrm{pH} 1.5$ (previously at $40^{\circ} \mathrm{C}$ for $40 \mathrm{~min}$ ) and at $\mathrm{pH} 4.5$ for one week, samples were analysed using HPLC analysis for $\mathrm{pH}$ stability evaluation. The samples where assayed by reversed-phase HPLC system with UV detection at $220 \mathrm{~nm}$ and $10 \mu \mathrm{L}$ loop. The mobile phase was a two-phases mixture of (A) acetonitrile/ trifuoracetic acid $0.1 \%$ in water MilliQ (10:90) and (B) acetonitrile/trifuoracetic acid $0.1 \%$ in water MilliQ (90:10). The gradient program used was $100 \%$ (A) from 0 to $1 \mathrm{~min}$; from 1 to $20 \mathrm{~min}$ a increasing gradient from 0 to $100 \%$ (B) with a constant flow rate of $1 \mathrm{~mL} / \mathrm{min}$.

An assay for the minimum quantity of Leu-Enk added to nanoparticles leads to determine the amount of Leu-Enk which is sufficient to fill up the nanoparticles and saturate the system. Leu-Enk in increasing concentrations were added to the medium of polymerization, and later Leu-Enk in the supernatant was determined by HPLC, previous separation of the nanoparticles from the supernatant by ultracentrifugation at $45 \times 10^{3} \mathrm{rpm}$ during $30 \mathrm{~min}$.

The methodology for Leu-Enk inclusion into the nanoparticles was carried out with two variations and is described in the fig. 2 . The first one consisted on the addition of Leu-Enk into the chitosan acidic solution before start of the polymerization with IBCA, called "time $=0$ hour" $(t=0 h)$. The second variation consisted on the addition of Leu-Enk one hour after the end of polymerization reaction, called "time $=1$ hour" $(t=1 h)$. This is in order to evaluate the quantity of Leu-Enk strictly encapsulated on the core of the nanoparticles and the quantity adsorpted at the surface of nanoparticles as well. a) time $=0 \mathrm{~h}$

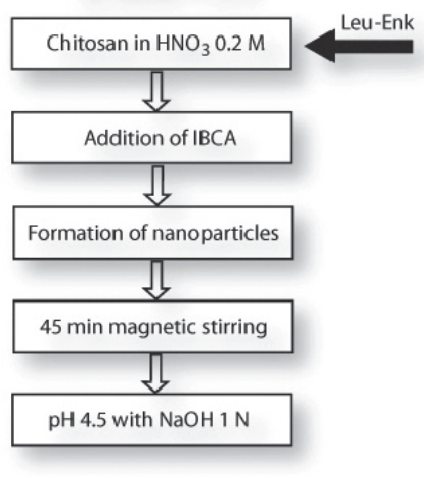

b) time $=1 \mathrm{~h}$

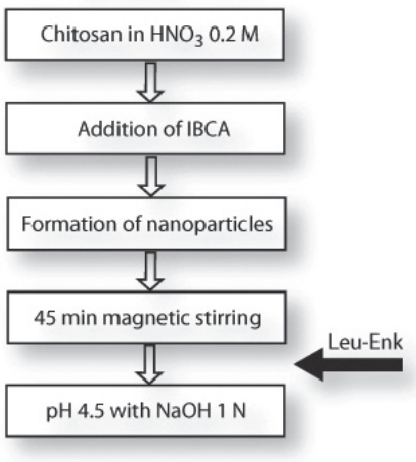

Figure 2. Two different methodologies for load of Leu-Enk: a) adding the drug before start the polymerization $(t=0 h), \mathrm{b})$ adding the Leu-Enk one hour after start the polymerization $(t=1 \mathrm{~h})$

Solubilization of the co-polymer nanoparticles for encapsulation yield determination: Bertholon et al. describe methodologies for IBCA/Dextran non-loaded nanoparticles solubilization using $\mathrm{NaOH} 1 \mathrm{~N}$ and dimethyl sulfoxide $(\mathrm{DMSO})^{18}$. This two agents were evaluated to solubilize IBCA/ChitoTBA nanoparticles loaded with Leu-Enk $\left(75 \mu \mathrm{gg}^{-1}\right)$ for later direct determination of the encapsulated drug released. The method of solubilization is described in fig. 3 .

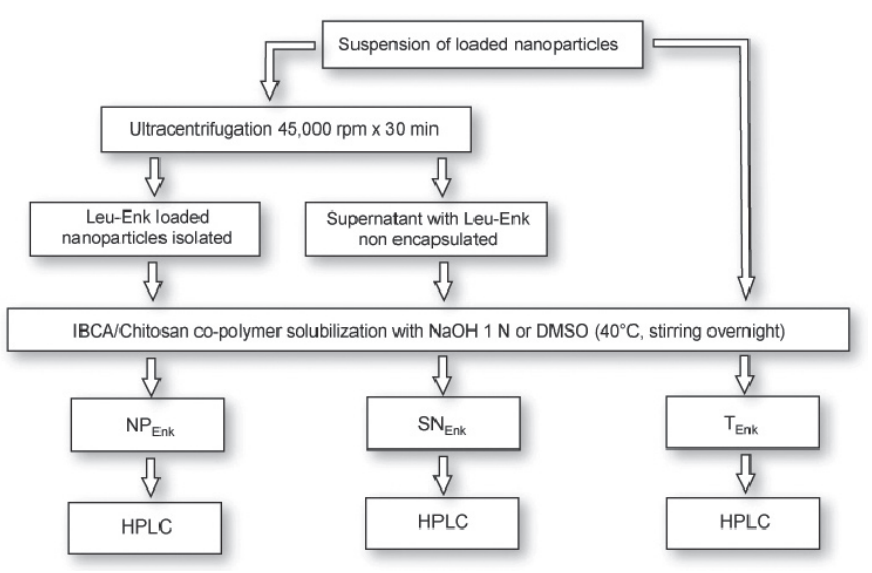

Figure 3. Solubilization of co-polymer nanoparticles for direct determination of loaded Leu-Enk by HPLC. $\mathrm{NP}_{\text {Enk }}$ is the drug encapsulated in the nanoparticles, $\mathrm{SN}_{\mathrm{Enk}}$ is the drug in the supernatant (non encapsulated), and $\mathrm{T}_{\text {Enk }}$ is the total drug both encapsulated and non encapsulated.

Yield of encapsulation and recovery assay: Leu-Enk recovery yield of the solubilization method it is necessary for assure the peptide integrity after the entire process of nanoparticle rupture for direct determination of the drug encapsulated. The yield of encapsulation (Y) was carried out adding a known Leu-Enk concentration $\left(75 \mu \mathrm{gg}^{-1}\right)$ in the nanoparticles formulation and was calculated according to the equation 1 :

$$
Y=\frac{N P_{E n k}}{T_{E n k}} .100
$$

Where $N P_{E n k}$ is the Leu-Enk concentration in loaded nanoparticles and $T_{E n k}$ is the total concentration of Leu-Enk in the complete nanoparticles suspension (encapsulated and non-encapsulated). The recovery yield of the solubilization method is gived by $T_{E n k}$. Loaded nanoparticles were isolated from the supernatant by using an ultracentrifugation at $45 \times 10^{3} \mathrm{rpm}$ during 30 $\min . N P_{E n k}$ and $T_{E n k}$ were obtained after total solubilization of nanoparticles 
with the $\mathrm{NaOH} 1 \mathrm{~N}$ or DMSO as shows in the fig. 3 and measured by HPLC in conditions previously described.

Physico-chemical characterization of the nanoparticles:

Determination of the $\zeta$ potential: The electric surface charge of the copolymer particles was deduced from the electrophoretic mobility of the particles measured by Laser Doppler Electrophoresis in a $\mathrm{NaCl} 1 \mathrm{mmol}^{-1}$ solution after suitable dilutions $(1 / 200 \mathrm{v} / \mathrm{v})$ for different nanoparticles suspensions in order to maintain a constant ionic strength ${ }^{19}$.

Nanoparticles morphology and size: The nanoparticle morphology was analysed by Scanning Electronic Microscopy (SEM) with nanoparticle suspensions diluted in MilliQ ${ }^{\circledR}$ water from $1 / 1$ to 1/10000. Nanoparticles size measurements were analysed by Transmission Electron Microscopy (TEM) by direct observation after staining with phosphotunguestic acid $1 \%(\mathrm{pH} \mathrm{7.4)}$ and the size measure is ensured using Adobe Photoshop ${ }^{\circledR}$ Software.

\section{Determination of thiol content in the nanoparticles:}

Iodine titration: The degree of modification of thiolated polymer and nanoparticles was analysed by iodine titration using soluble starch, measured by spectrophotometry at $560 \mathrm{~nm}$ against a calibration curve of L-cystein $(0.04-$ $\left.0.124 \mu \mathrm{mol} \mathrm{mL}^{-1}\right)$, as described by Bravo-Osuna et $a l^{20}$.

Elemental analysis: The total amount of sulphur in both thiolated polymers and nanoparticles was determined by elemental analysis. Samples of 10 $\mathrm{mg}$ were burned at $1350^{\circ} \mathrm{C}$ over oxygen flux and the detection of $\mathrm{SO}_{2}$ was performed by infrared measurements

\section{RESULTS AND DISCUSSION}

\section{Chitosan modifications and characterization:}

From commercial chitosan, three average molecular weights were obtained after depolymerization: $30,000 \mathrm{~g} \mathrm{~mol}^{-1}, 85,000 \mathrm{~g} \mathrm{~mol}^{-1}$ and 145,000 $\mathrm{g} \mathrm{mol}^{-1}$ (called Chito30, Chito 85 and Chito145 respectively) measured by capillary viscosity. ${ }^{1}$ NMR showed no structural change and acceptable decrease in the percentage of deacetylation, with values about 60,71 and $89 \%$ for Chito30, Chito85 and Chito145 respectively. The total sulphur in depolymerized modified chitosan (Table 1) showed no significant variation among them. The reduced thiol groups content in modified chitosan decreased according the chitosan molecular weight probably due to differences in deacetylation degree, because the thiol group from the Traut's reagent should be included on the free amino groups of the chitosan chain (fig. 4).

Table 1. Total sulphur and thiol group determination in depolymerized chitosanTBA and IBCA/ChitosanTBA nanoparticules elaborated by anionic emulsion polymerization.

\begin{tabular}{|l|c|c|}
\hline \multicolumn{1}{|c|}{ Depolymerized modified chitosan } & $\begin{array}{c}\text { Total S \% } \\
(\mathrm{w} / \mathrm{w})^{(\mathrm{a})}\end{array}$ & $\begin{array}{c}-\mathrm{SH} \mu \mathrm{mol} \\
\mathrm{g}^{-1(\mathrm{~b})}\end{array}$ \\
\hline Chito30-TBA & 4.7 & $30.1 \pm 4.3$ \\
\hline Chito85-TBA & 5.1 & $15.2 \pm 3.2$ \\
\hline Chito145-TBA & 4.8 & $4.1 \pm 0.5$ \\
\hline \multicolumn{1}{|c|}{ IBCA/ChitosanTBA nanoparticles } & & \\
\hline Chito30 75\% / Chito30-TBA 25\% & 0.34 & $0.11 \pm 0.03$ \\
\hline Chito85 75\% / Chito85-TBA 25\% & 0.35 & $0.08 \pm 0.02$ \\
\hline Chito145 75\% / Chito145-TBA 25\% & 0.59 & $0.04 \pm 0.02$ \\
\hline
\end{tabular}

(a) Elemental analysis of total sulphur $(n=1)$.

(b) Iodine titration of thiol groups: calibration curve between 0.04 and $0.124 \mu \mathrm{mol} \mathrm{mL}^{-1}$ of L-cystein $\mathrm{HCl}$ (intercept mean $=0.225$; slope mean $=-0.002$; $\left.r^{2}=0.989\right)(n=3)$

\section{Physico-chemical characterization of nanoparticles:}

The resulting dispersion made by anionic emulsion polymerization is composed by a isobutylcyanoacrylate/thiolated chitosan copolymer (fig. 4) which spontaneously auto-associates to form nanoparticles (fig. 5). The polymer particles are likely stabilized by the hydrophilic polysaccharide moiety as already suggested Passirani et $a l^{21}$.

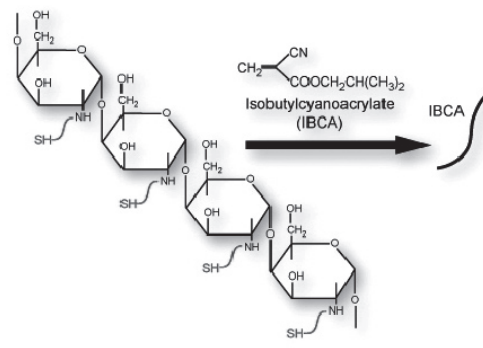

ChitosanTBA

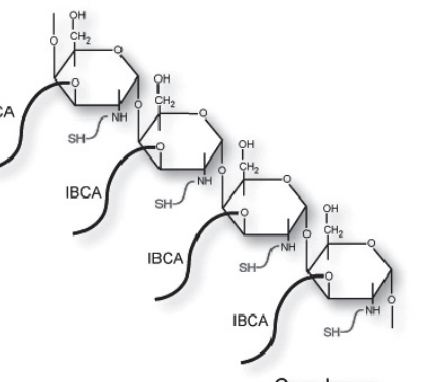

Copolymer IBCA/ChitosanTBA
Figure 4. Mechanism of anionic emulsion polymerization of IBCA in the presence of Chitosan/ChitosanTBA in acidic media.

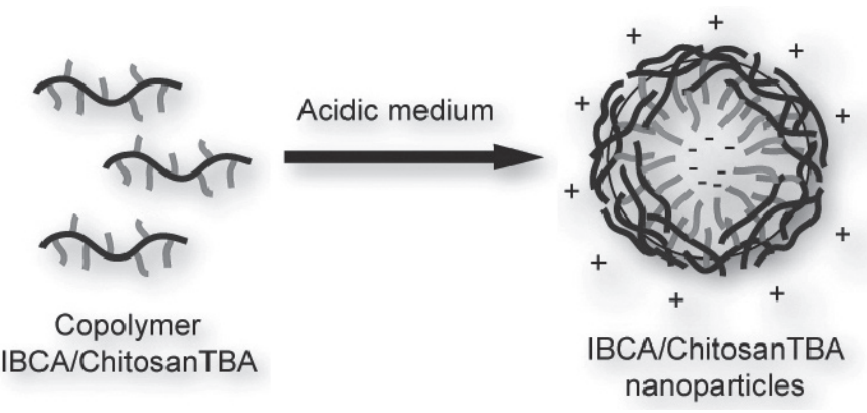

Figure 5. Formation of IBCA/ChitosanTBA nanoparticles in acidic medium from amphiphilic co-polymers.

The morphologic characteristics of the different nanoparticles obtained are gathered in table 2. Results obtained from the study of the electrophoretic mobility of the nanoparticles offered positive values of $\zeta$ potential for all formulations, indicating that cationic polysaccharide was located at the surface of the nanoparticles (fig. 5). The polysaccharide coating completely masked the negative surface charge values characteristic of non-coated IBCA nanoparticles as well described by previous works ${ }^{22}$.

Table 2. Nanoparticles characterization of different formulations elaborated by anionic emulsion polymerization.

\begin{tabular}{|l|c|c|}
\hline \multicolumn{1}{|c|}{ IBCA/ChitosanTBA nanoparticles } & D (nm) ${ }^{(\mathrm{a})}$ & $\begin{array}{c}\zeta \text { potential } \\
(\mathrm{mV})^{(\mathrm{b})}\end{array}$ \\
\hline Chito30 75\% / Chito30-TBA 25\% & $28.4 \pm 3.5$ & $+38.5 \pm 0.7$ \\
\hline Chito85 75\% / Chito85-TBA 25\% & $29.4 \pm 4.2$ & $+41.8 \pm 0.9$ \\
\hline Chito145 75\% / Chito145-TBA 25\% & $46.8 \pm 3.4$ & $+53.1 \pm 0.7$ \\
\hline
\end{tabular}

(a) Mean diameter $(D)(n=100)$ $(n=3)$

(b) Surface charge determination ( $\zeta$ potential) in $\mathrm{NaCl} 1 \mathrm{mmol} \cdot \mathrm{L}^{-1} \mathrm{pH} 6.7$

Small nanoparticles about 28 to $46 \mathrm{~nm}$ diameter were obtained. The size of the nanoparticles mainly depend on the molecular weight of the chitosan used considering the series of experiments performed with thiolated-chitosan. It can be observed that the lower the molecular weight, the smaller the nanoparticles size. Nanoparticles with small size have great importance for the stability of the polymer particles and leads to a high exposition of thiols groups at the surface of nanoparticles, and better mucoadhesion is expected when oral administration is perfomed.

TEM technique allowed the isolation and easy observation of spherical and very well individualized particles (fig. 6). As can be noticed, in all cases spherical particles were obtained in the nano-size range. 

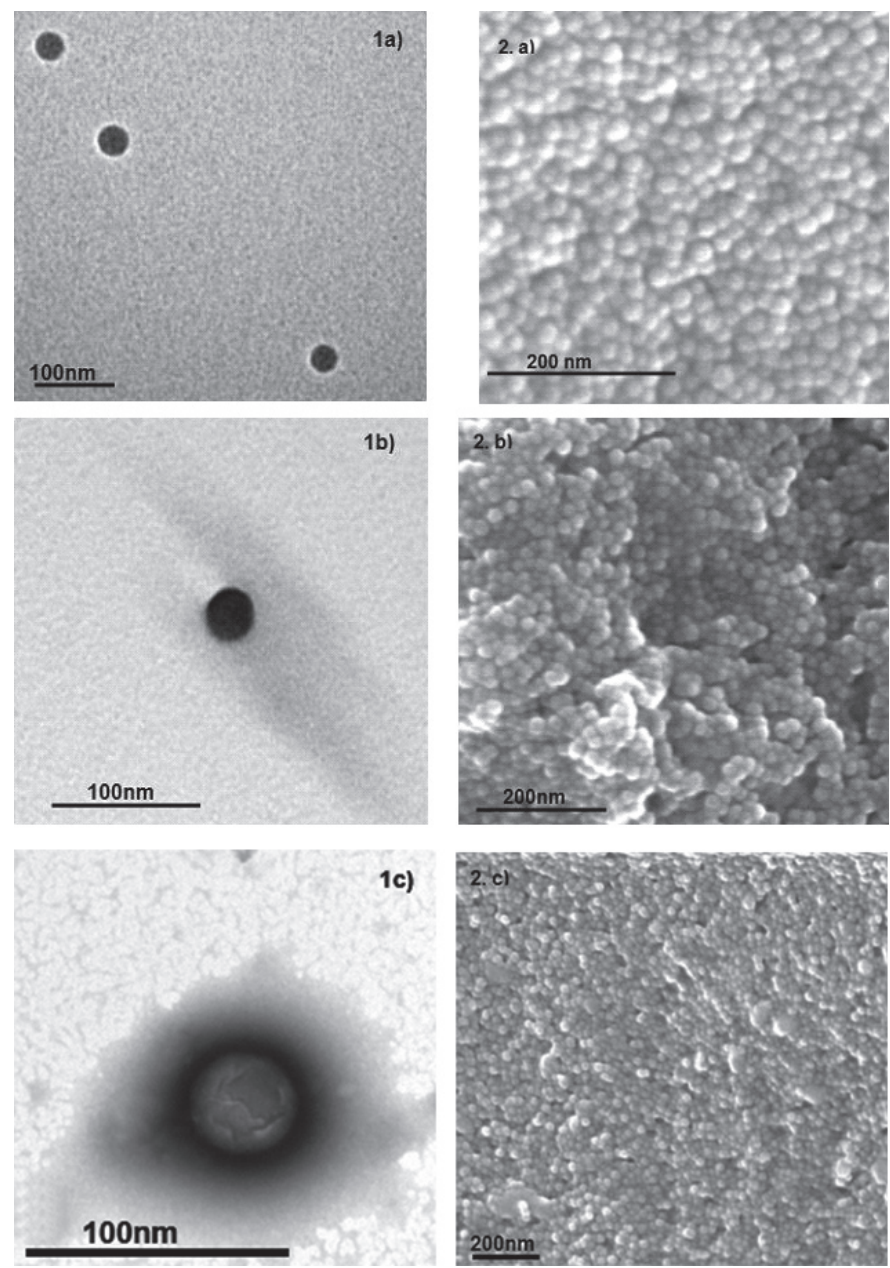

Figure 6. (1) Transmission Electron Microphotographs (TEM) and (2) Scanning Electron Microphotographs (SEM) of nanoparticles elaborated with ChitoTBA 30 (a), ChitoTBA 85 (b) and ChitoTBA 145 (c) following anionic emulsion polymerization.

Total sulphur and thiol groups determination: The values of total amount of sulphur contained in the nanoparticles formulations showed no relationships between chitosan molecular weight. The thiol groups is reduced along chitosan molecular weight is incresed, probably due to thiol groups amount in its respective modified chitosan (Table 1).

3. Inclusion of Leu-Enkephalin into the nanoparticles:

Stability of Leu-Enk at different pH: The Leu-Enk stability at different $\mathrm{pH}$ and temperatures were studied. First, $\mathrm{pH} 1.5$ at $40^{\circ} \mathrm{C}$ because the anionic emulsion polymerization occurs at this $\mathrm{pH}$, and $\mathrm{pH} 4.5$ because after the end of the polymerization reaction, the $\mathrm{pH}$ of the solution was rised to 4.5 for store. Results indicate that recovery of Leu-Enk was $101.7 \% \pm 0.7(\mathrm{n}=3)$ for $\mathrm{pH} 1.5$ and $101.8 \pm 0.9(\mathrm{n}=3)$ for $\mathrm{pH} 4.5$, indicating a satisfactory stability for Leu-Enk at these polymerization and store conditions.

Filled-up limit of Leu-Enkephalin encapsulation into nanoparticles: Fig. 7 shows that there is a direct response of Leu-Enk found in the supernatant when peptide concentration is increased in the nanoparticles formulation. The inflection point is approximately $10 \mu \mathrm{g} \cdot \mathrm{g}^{-1}$ of Leu-Enk added, and after that point, Leu-Enk concentration start to increased directly in the supernatant, while the Leu-Enk encapsulated into the nanoparticles remains constant, that can be due to a saturation of the capacity of nanoparticles.

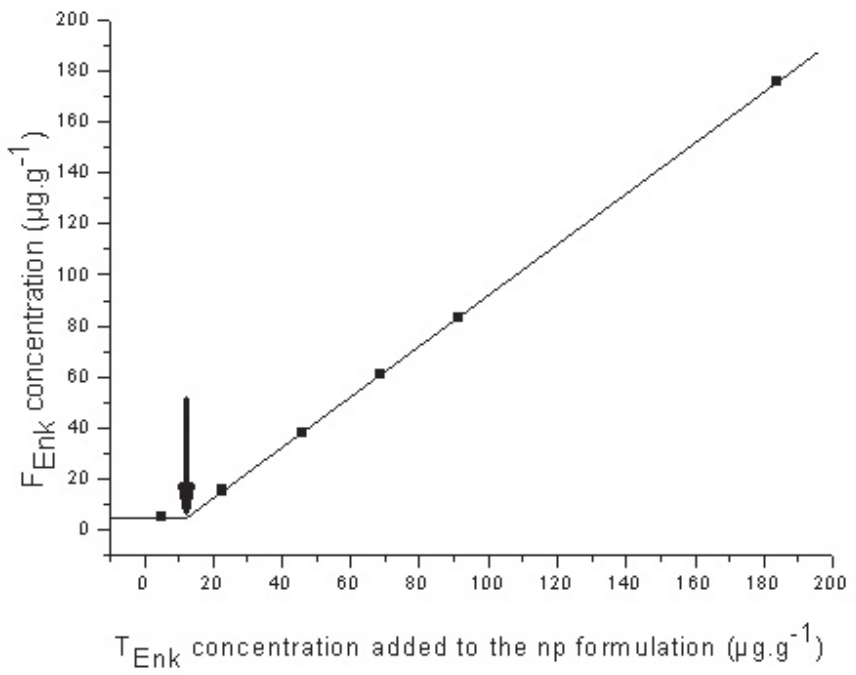

Figure 7. Determination filled-up limit of Leu-Enkephalin in nanoparticles IBCA/Chito30-TBA using anionic emulsion polymerization $\left(\mathrm{SN}_{\mathrm{Enk}}=\mathrm{Leu}-\mathrm{Enk}\right.$ concentration in the supernatant) Concentrations obtained using HPLC analysis (intercept mean $=-15050$; slope mean $=46671 ; \mathrm{r}^{2}=0.999$; repeatability $=1.92 \%$; intermediate precision $=1.45 \%$ )

4. Solubilization method for amphiphilic co-polymer nanoparticles and recovery yields for total Leu-Enk in the formulation:

In most of the cases, indirect methods of load drug in nanoparticles systems are developted, this is, the loaded drug is measured by the difference between the total drug added to the system, and the drug in the supernatant, been this last one, directly measured. In this study, we formulate a direct load drug determination method. First, it is necessary to develop a solubilization method for nanoparticles, so the loaded drug can be released without destruction, and then measured directly. In addition, drug in the supernatant and total drug added to the system must be measured directly as it shows the fig. 3 for recovery yield determination of the method.

Co-polymer solubilization methods with $\mathrm{NaOH} 1 \mathrm{~N}$ and DMSO were evaluated for drug load determination. The table 3 shows that $\mathrm{NaOH}$ presents low total Leu-Enk recovery yields reached by direct determination of $T_{\text {in }}$ in the range of $64 \%$. But in the case of DMSO, yields of recovery higher than $96 \%$ are reached in $\mathrm{T}_{\text {Enk }}$, and a logical correlation between the Leu-Enk in the supernatant and the Leu-Enk released from nanoparticles is obtained for DMSO, that give us a more reliable co-polymer solubilization method, and no Leu-Enk destruction along the procedure is deduced.

Table 3. Determination of the yield of encapsulation (\%) of Leu-Enk (75 $\mu \mathrm{gg}^{-1}$ load concentration) in IBCA/Chito-TBA nanoparticles obtained by anionic emulsion polymerization using $\mathrm{NaOH} 1 \mathrm{~N}$ and DMSO for nanoparticles rupture $(n=1)$.

\begin{tabular}{|c|c|c|c|c|c|c|}
\hline \multirow{2}{*}{$\begin{array}{c}\text { IBCA/Chitosan } \\
\text { nanoparticles }\end{array}$} & \multicolumn{3}{|c|}{$\mathrm{NaOH} 1 \mathrm{~N}$} & \multicolumn{3}{c|}{ DMSO } \\
\cline { 2 - 7 } & $\mathrm{SN}_{\text {Enk }}$ & $\mathrm{NP}_{\text {Enk }}$ & $\%_{\mathrm{T}}$ & $\begin{array}{c}\% \\
\mathrm{SN}_{\text {Enk }}\end{array}$ & $\begin{array}{c}\% \\
\mathrm{NP}_{\text {Enk }}\end{array}$ & $\begin{array}{c}\% \\
\mathrm{~T}_{\text {Enk }}\end{array}$ \\
\hline $\begin{array}{c}\text { Chito30 75\%/ } \\
\text { Chito30-TBA } \\
\mathbf{2 5 \%}\end{array}$ & 32.2 & 33.3 & 63.4 & 54.1 & 46.8 & 98.7 \\
\hline $\begin{array}{c}\text { Chito85 75\%/ } \\
\text { Chito85-TBA } \\
\mathbf{2 5 \%}\end{array}$ & 35.0 & 27.4 & 65.7 & 60.4 & 42.4 & 96.1 \\
\hline $\begin{array}{c}\text { Chito145 75\%/ } \\
\text { Chito145-TBA } \\
\mathbf{2 5 \%}\end{array}$ & 41.2 & 13.6 & 64.9 & 61.0 & 44.8 & 98.1 \\
\hline
\end{tabular}

$S N_{E n k}=$ Leu-Enk percentage in the supernatant, $N P_{E n k}=$ Leu-Enk in loaded nanoparticles, and $T_{F}=$ total concentration of Leu-Enk in the nanoparticles suspension directly determined respectively. Concentrations of Leu-Enk obtained using HPLC analysis (intercept mean $=-15050$; slope mean $=46671$; $r^{2}=0.999$; repeatability $=1.92 \%$; intermediate precision $=1.45 \%$ ). 


\section{Determination of encapsulation yield:}

Once the optimal co-polymer solubilization method and filled-up limit of Leu-Enk in nanoparticles are determinated, yield of encapsulation was carried out for both methods of Leu-Enk inclusion: adding the drug before polymerization $(t=0 h)$, and adding one hour after the start of the polymerization $(t=1 \mathrm{~h})$ as seen on fig. 2.

Table 4 shows that yield of encapsulation of $42-46 \%(0,427-1,770 \mathrm{ng}$ Leu-Enk/nanoparticle) was achieved with Leu-Enk inclusion at $t=0 h$, that results encapsulation into the core of nanoparticles and surface adsorption of Leu-Enk outside nanoparticles. Yield of encapsulation of 25-38\% (0,388-1.290 ng LeuEnk/nanoparticle) was obtained when Leu-Enk is added at $t=1 \mathrm{~h}$, probably due only to adsorpted Leu-Enk in the surface of nanoparticles. Kinetics of formation in previous studies of IBCA/dextran nanoparticles show that after the first 15 minutes no longer nanoparticles are formated for anionic polymerization ${ }^{23}$, so most of the Leu-Enk is encapsulated fast in the first minutes of polymerization, so adding the Leu-Enk one hour after start the polymerization, a great porcentage of LeuEnk is only adsorpted in the surface at $t=1 \mathrm{~h}$, resulting theoretically $0.039-0.480 \mathrm{ng}$ Leu-Enk/nanoparticle strictly encapsulated inside the nanoparticles.

Table 4. Yield of Leu-Enk of Leu-Enk found in supernatant $\left(\% \mathrm{SN}_{\mathrm{Enk}}\right)$, encapsulated in nanoparticles $\left(\% \mathrm{NP}_{\mathrm{Enk}}\right)$ and in the total formulation $\left(\% \mathrm{~T}_{\mathrm{Enk}}\right)$ at different Leu-Enk inclusion times. Co-polymer solubilization performed with DMSO $40^{\circ} \mathrm{C}$ under continuous stirring overnight $(\mathrm{n}=1)$.

\begin{tabular}{|c|c|c|c|c|c|c|c|c|}
\hline IBCA/ChitoTBA nanoparticles & \multicolumn{4}{|c|}{ Inclusion of Leu-Enk ${ }^{\mathrm{b}}$ at $t=0 h$} & \multicolumn{4}{|c|}{ Inclusion of Leu-Enk ${ }^{\mathrm{b}}$ at $t=1 \mathrm{~h}$} \\
\hline Chito30 75\%/Chito30-TBA 25\% & 54.1 & 46.8 & 98.7 & 0.427 & 68.9 & 38.3 & 98.9 & 0.388 \\
\hline Chito85 75\%/Chito85-TBA 25\% & 60.4 & 42.4 & 96.1 & 0.419 & 70.3 & 25.9 & 101.0 & 0.292 \\
\hline Chito145 75\%/Chito145-TBA 25\% & 61.0 & 44.8 & 98.1 & 1.770 & 76.8 & 27.7 & 102.9 & 1.290 \\
\hline
\end{tabular}

(a) $n g$ of Leu-Enk by one nanoparticle. Concentrations of Leu-Enk obtained using HPLC analysis (intercept mean=-15050; slope mean=46671; $r^{2}=0.999$; repeatability $=1.92 \%$; intermediate precision $=1.45 \%)$.

(b) Load concentration: $75 \mu \mathrm{gg}^{-1}$

\section{CONCLUSIONS}

Isobutylcyanoacrylate nanoparticles coated with thiolated chitosan were prepared using anionic emulsion polymerization. Nanoparticles in the order of 28-46 nm were obtained, and expected thiolation degree was found in the formulations. For direct encapsulation yield determination, nanoparticle copolymer solubilization method with $\mathrm{NaOH} 1 \mathrm{~N}$ described in previous works for non-loaded nanoparticles, can not be used when formulation of loaded nanoparticles with Leu-Enk is performed. Several loss of peptide is found when nanoparticles hydrolysis with $\mathrm{NaOH} 1 \mathrm{~N}$ is used for solubilization of IBCA/ ChitosanTBA co-polymer, but no loss of peptide is found when DMSO is used for co-polymer solubilization, being a usefull tool for direct quantification of encapsulated Leu-Enk in IBCA/ChitosanTBA nanoparticle systems, resulting Leu-Enk recovery yields higher than $96 \%$ for this method. The encapsulation yields of Leu-Enk were satisfactory by anionic emulsion polymerization, achieving $42-47 \%$ when peptid is added before the start of the polymerization (direct core encapsulation and surface adsorption), and 26-38\% when is added after the polymerization (only adsorption mechanism).

\section{ACKNOWLEDGEMENTS}

Authors want to thank Dr. K. Broadley from Loctite (Dublin, Ireland) for his kindness in providing the isobutylcyanoacrylate monomer. Authors want also to thank the Department of Organic Chemistry (Biocis UMR CNRS 8076), Faculty of Pharmacy, University Paris-XI (Chatenay-Malabry, France) for their help in the synthesis of 2- iminothiolane and the "Service central d'analyse du CNRS" (Vernaison, France) for the elemental analysis of thiolated polymers. Authors want also to thank Irene Bravo-Osuna, Madeleine Besnard and Alexia Aspe. Thanks to Audrey Valette (CNRS CECM Vitry-surSeine, France) and Jeril Degrouard (UMR CNRS 8080, Orsay, France), for their help in the characterization of nanoparticles by SEM and TEM. Finally, Víctor Campos wants to thank to CONICYT-Chile for supporting his Magister in Pharmaceutical Sciences with a two-years grant.

\section{REFERENCES}

1. I. Bravo-Osuna, C. Vauthier, H. Chacun, G. Ponchel, Eur. J. Pharm. Biopharm. 69 (2), 436-444 (2008).

2. A. Bernkop-Schnürch, D. Guggi, Y. Pinter, J. Control. Rel. 94, 177-186 (2004).

3. M. Aboubakar, P. Couvreur, H. Pinto-Alphandary, B. Gouriton, B. Lacour, R. Farinotti, F. Puisieux, C. Vauthier, Drug Dev. Res. 49, 109-117 (2000).
4. Ying-Shu Quan, Takuya Fujita, Daichi Tohara, Miwako Tsuji, Makoto Kohyama, Akira Yamamoto, Life Sciences, Vol 64, №14, 1243-1252 (1999).

5. G. Pauletti, F. Okumu, R. Borchardt, Pharm. Res. Vol 14, No.2, 164-168 (1997).

6. A. Bernkop-Schnürch, M. Hornof, D. Guggi. Eur. J. Pharm. Biopharm. 57, 9-17 (2004).

7. A. Bernkop-Schnürch, Y. Pinter, D. Guggi, H. Kahlbacher, G. Schöffmann, M. Schuh, I. Schmerold, M. Dorly, M. D’Antonio, P. Esposito, C. Huck. J. Control. Rel. 106, 26-33 (2005).

8. A. Bernkop-Schnürch. Adv. Drug Del. Rev. 57, 1569-1582 (2005).

9. A. Bernkop-Schnürch, A. Krauland, V. Leitner, T. Palmberger. Eur. J. Pharm. Biopharm. 58, 253-263 (2004).

10. I. Bertholon, D. Labarre, C. Vauthier. Polymer 46, 1407-1415 (2005).

11. I. Bertholon, C. Vauthier, D. Labarre. Pharm. Res. 23 (6), 1313-1323 (2006).

12. C. Chauvierre, M. Marden, C. Vauthier, D. Labarre, P. Couvreur, L. Leclerc, Biomaterials 25, 3081-3086 (2004).

13. M. Huang, E. Khor, L.Y. Lim, Pharm Res. 21(2), 344-353 (2004).

14. A. Bernkop-Schnürch, M. Hornof, T. Zoidl. Int. J. Pharm. 260, 229-237 (2003).

15. C. Chauvierre, D. Labarre, P. Couvreur, C. Vauthier, Macromol. 36, 60186027 (2003).

16. I. Bravo-Osuna, G. Ponchel, C. Vauthier, Eur. J. Pharm. Sci. 30 (2), $143-$ 154 (2007).

17. C. Chauvierre, D. Labarre, P. Couvreur, C. Vauthier, Pharm Res 20 (11) 1786-1793 (2003).

18. I. Bertholon, S. Lesieur, D. Labarre, M. Besnard, C. Vauthier. Macromol. 39, 3559-3567 (2006).

19. M.A. Arangoa, G. Ponchel, A. M. Orecchioni, M. J. Renedo, D. Duchene, J. M. Irache, Eur. J. Pharm. Sci. 11 (4), 333-341 (2000).

20. I. Bravo-Osuna, T. Schmitz, A. Bernkop-Schnürch, C. Vauthier, G. Ponchel, Int. J. Pharm. 316, 170-175 (2006)

21. C. Passirani, G. Barratt, J.P. Devissaguet, D. Labarre, Life Sci. 62 (8), $775-$ 785 (1998).

22. M. T. Peracchia, C. Vauthier, C. Passirani, P. Couvreur, D. Labarre, Life Sci. 61 (7), 749-761 (1997).

23. C. Chauvierre, D. Labarre, P. Couvreur, C. Vauthier, J. Nano. Res. 5, 365371 (2003) 